\title{
Clinical outcome of endonasal endoscopic prelacrimal approach in managing different maxillary pathologies
}

\author{
Yu Hsuan Lin ${ }^{1}$, Wei-Chih Chen ${ }^{\text {Corresp. } 2}$ \\ ${ }^{1}$ Department of Otolaryngology, Kaohsiung Veterans General Hospital, Kaohsiung, Taiwan \\ 2 Chang Jung University, Kaohsiung, Taiwan \\ Corresponding Author: Wei-Chih Chen \\ Email address: jarva@adm.cgmh.org.tw
}

Background. The aim of the study was to evaluate the treatment outcomes of endoscopic prelacrimal recess approaches (EPLAs) in managing different sinus pathologies, analyzing associated adverse events and post-treatment quality-of-life.

Methods. We enrolled 21 consecutive patients (22 lesions) who received endoscopic sinus surgical procedures with EPLAs in two tertiary medical institutes between 2015 and 2018. Quality-of-life and selfrated symptom severity data were collected using the 22-item Sino-Nasal Outcomes Test (SNOT-22) and 10-point visual analogue scales (VAS), respectively.

Results. Twenty-one patients (mean age [standard deviation] 51.7 [14.5] years; 16(76.2\%) male) were followed up for 12.7 months. The most common symptoms were nasal discharge and nasal airway obstructions. Nine lesions (40.9\%) were sinonasal papilloma's, 7 lesions were other types of neoplasms (31.8\%; 5 benign and 2 malignant), 2 were trauma-related (9.1\%), and 4 inflammatory diseases (18.2\%). Patients with non-papilloma lesions had higher presurgical SNOT-22 than those with papillomas ( $p$ value $=0.021$ ). After EPLAs, non-papilloma patients had significant improvements in SNOT-22 and VAS ( $p$ values $=0.012 \& 0.012$, respectively), while those with papillomas had only marginally significant improvements in VAS ( $p$-value $=0.061$ ). The most common adverse events was temporary cheek/tooth numbness $(n=11)$, and patients with sinonasal papillomas were more likely to have post-treatment complications than those with other disease entities.

Conclusions. Endoscopic PLAs were found to effectively manage various sinus diseases. Short-term lifequality improvements were promising. Future large-scale studies with longer follow-up periods are recommended. 
1 Clinical Outcome of Endonasal Endoscopic Prelacrimal Approach in Managing

2 Different Maxillary Pathologies

3

4 Type of article: Research Article

5

6 Yu-Hsuan Lin, MD ${ }^{1,2}$, Wei-Chih Chen, $\mathrm{MD}^{3}$

7 1. Department of Otolaryngology, Head and Neck Surgery, Kaohsiung Veterans General

8 Hospital, Kaohsiung, Taiwan.

9 2. Department of Otolaryngology, Head and Neck surgery, National Defense Medical Center,

10 Taipei, Taiwan.

11 3. Department of Otolaryngology, Kaohsiung Chang Gung Memorial Hospital and Chang Gung University College of Medicine, Kaohsiung, Taiwan

Address correspondence to:

Wei-Chih Chen, MD

17 Department of Otolaryngology, Kaohsiung Chang Gung Memorial Hospital

E-mail address: jarva@adm.cgmh.org.tw

Tel: +886-7-7317123 ext. 2533; Fax: +886-7-7313855

20

21 Numbers: Figures-2

\section{Competing interests}


23 None of the authors declare any conflict of interest, financial or otherwise.

\section{Authors' contributions}

25 Conception and design: L-YH, C-WC

26 Collection and assembly of data: L-YH, C-WC

27 Data analysis and interpretation: L-YH, C-WC

28 Manuscript writing: L-YH, C-WC

29 Final approval of manuscript: L-YH, C-WC

30 Funding: None

31 This manuscript is original and it, or any part of it, has not been previously

32 published. It is not under consideration for publication elsewhere. 


\section{Abstract}

\section{Background}

The aim of the study was to evaluate the treatment outcomes of endoscopic prelacrimal recess approaches (EPLAs) in managing different sinus pathologies, analyzing associated adverse events and post-treatment quality-of-life.

\section{Methods}

We enrolled 21 consecutive patients (22 lesions) who received endoscopic sinus surgical procedures with EPLAs in two tertiary medical institutes between 2015 and 2018. Quality-of-life and self-rated symptom severity data were collected using the 22-item Sino-Nasal Outcomes Test (SNOT-22) and 10-point visual analogue scales (VAS), respectively.

\section{Results}

Twenty-one patients (mean age [standard deviation] 51.7 [14.5] years; 16 (76.2\%) male) were followed up for 12.7 months. The most common symptoms were nasal discharge and nasal airway obstructions. Nine lesions (40.9\%) were sinonasal papilloma's, 7 lesions were other types of neoplasms (31.8\%; 5 benign and 2 malignant), 2 were trauma-related (9.1\%), and 4 inflammatory diseases (18.2\%). Patients with non-papilloma lesions had higher presurgical SNOT-22 than those with papillomas ( $p$-value=0.021). After EPLAs, non-papilloma patients had significant improvements in SNOT-22 and VAS (p-values=0.012\&0.012, respectively), while those with papillomas had only marginally significant improvements in VAS ( $p$-value=0.061). The most common adverse events were temporary cheek/tooth numbness $(n=11)$. Patients with sinonasal papillomas were more likely to have post-treatment complications than those with other disease entities. 


\section{Conclusions}

58 Endoscopic PLAs were found to effectively manage various sinus diseases. Short-term life-

59 quality improvements have been promising. However, future large-scale studies with longer

60 follow-up periods are recommended.

61

62 Key words

63 Prelacrimal recess, endoscopic prelacrimal recess approach, papilloma, 22-item Sino-Nasal 64 Outcomes Test, life quality.

65 
66

67

68

69

70

71

72

73

74

75

76

77

\section{Introduction}

Maxillary sinus is the largest of the paranasal sinuses (Tomenzoli et al.,2014). It is bordered posteriorly by the pterygopalatine fossa and infratemporal fossa, inferiorly by the alveolar process and superiorly by the orbital floor (Tomenzoli et al.,2014). Unilateral maxillary occupying lesions encompass a broad array of pathologies, predominantly sinusitis, followed by sinus cyst, and then benign neoplasms (mostly sinonsal papillomas) (Coleman et al.,2005). Because the maxillary sinus is the most frequent site of sinus pathologies, mere ostium opening by endoscopic approach may not adequately address all the problems (Kennedy \& Adappa, 2011).

Most diseases of the maxillary sinus can be managed by endoscope, which is used mostly to perform standard middle meatal antrostomy (MMA) (Kennedy \& Adappa, 2011). For benign lesions such as inverted papillomas, endoscopic medial maxillectomies are often performed for better operating field and management of the tumor attachment site (Turri-Zanoni et al.,2017; Wormald et al., 2003). While it is almost impossible to resolve every detail of the disease with conservative approach, more extended procedures are performed at increased risk of surgeryrelated morbidities (Turri-Zanoni et al.,2017; Wormald et al.,2003; Lombardi et al.,2011; Bertazzoni et al.,2017). Nakamaru (2010) introduced the surgical creation of a corridor made by breaking a hole through the medial wall of prelacrimal recess (PLR), which is usually one of the most difficult positions to manipulate during standard MMA. This surgical corridor can provide an unobstructed view of almost any aspect of the maxillary inner linings. This improved visualization of the operating field can reduce the risks associated with endoscopic procedures (Wormald et al.,2003; Lombardi et al.,2011; Bertazzoni et al.,2017). 
90

91

93

Zhou et al. have promulgated the use of the endoscopic prelacrimal recess approach (EPLA) to manage a variety of pathologies of the maxillary sinus and deep areas of the skull base (Zhou et al.,2013; Zhou et al.,2016). The use of the EPLA has been reported to provide results comparable to those of conventional external procedures (Lee et al.,2019). However, its application has mostly been focused on the removal of neoplasms and no studies have been performed to assess post-treatment life-quality following EPLA (Lee et al.,2019; Zhou et al.,2018; Yu et al., 2018; Lin, Lin \& Yeh,2018). Therefore, in this study, we retrospectively reviewed the clinical applications of endoscopic prelacrimal recess approach in the surgical treatment of different maxillary pathologies. Patients were followed up to assess functional treatment outcomes (patient-reported overall health and symptom severity) as well as adverse events and need for revision surgery.

\section{Material and Methods}

\section{Study Design and Patient Eligibility}

We identified 21 adult patients receiving surgical procedures utilizing the endonasal endoscopic prelacrimcal recess approach (EPLA) from February 2015 to October 2018 in 2 tertiary care referral centers retrospectively. These patients may have also had additional endoscopic sinus surgeries or deviated septum corrections when deemed necessary by a surgeon based on pathology, extent of disease, or a patient's specific complaints prior to surgery. All patients enrolled in this study were recommended EPLAs because their diseases had been considered too difficult to treat using standard middle meatal antrostomy alone. The protocol of this study was approved by the institutional review board of Kaohsiung Chang Gung Memorial Hospital (IRB No.: 201900695B0). Written consents of the participants are not needed because of the retrospective nature of our study design. 
114 All patients received high-resolution sinus computed tomography scans (1.0-cm slices in the

115 axial view) to assess degree of inflammation. Sinus disease was scored based on modified-Lund-

116 Mackay scores (Snidvongs et al.,2014) of the diseased side and further categorized using the

117 Krous staging system (Krouse,2000) or American Joint Committee on Cancer (AJCC) TNM

118 classifications, depending on whether they were sinonasal papillomas or malignancies.

119 Prelacrimal recess space was measured as follows. Antero-posterior (AP) diameter was first

120 defined as the distance from pyriform aperture to the nasolacrimal duct (NLD) by identifying

121 the inferior-most aspect of NLD on coronal view and then transposing that to axial view. The

122 height of medial PLR was measured starting at the level of the superior-most aspect of the NLD

123 to the nasal floor, as described in Kashlan \& Craig (2018). Other demographic data including age,

124 gender, initial symptoms and their durations, image findings and known surgical history of each

125 patient were also collected from each patient prior to surgery. After surgery, patients received

126 a series of follow-up examinations in 3 to 6 visits spaced one to four weeks apart, depending on

127 endoscopic findings and specific needs of the patient. Daily saline irrigation and intranasal

128 corticosteroid spray were performed for post-treatment cares. Short-term oral medications

129 were prescribed only for symptomatic patients. Revision surgery was performed if disease

130 recurred.

131

132 Outcome Assessment

133 We assessed interval changes in patient-reported outcomes between initial baseline

134 evaluations and the last follow-up date. Functional outcome measurements included self-rated

135 visual analog scale for current overall health ( 0 being worst imaginable and 10 as best

136 imaginable) and self-rated 22-item Sino-Nasal Outcome Test (SNOT-22) to assess sinonasal

137 symptom severity (DeConde et al.,2015). Other outcomes were incidence and characterization 
138 of treatment-associated adverse events and need for revision surgery. Follow-up time was

139 defined as the time between the first visit with an otolaryngologist for diagnosis to the last

140 documented follow-up date.

141

142 Surgical technique

143 All surgical procedures were performed with patients under general anesthesia. EPLA was

144 performed before the other procedures for patients scheduled to receive endoscopic

145 prelacrimal recess approach for maxillary sinus lesions. To perform these procedures using the

146 prelacrimal recess approach, we first administered local anesthesia to the axilla of inferior

147 turbinate using a 4-mm, 18-cm length zero degree rod-lens endoscope (Karl Storz Endoscopy,

148 Tuttlingen, Germany). A 15-blade was used to create a curvilinear incision from the lateral nasal

149 cavity to the nasal floor, pathway crossing just anteriorly to the head of inferior turbinate. The

150 inferior turbinate-nasolacrimal duct (IT-NLD) flap was then elevated subperiosteally by a

151 suction elevator pulling it toward the mucosal portion of the naso-lacrimal duct and placed

152 medially. Using a 4-mm chisel to remove the head of inferior concha bone and part of frontal

153 process of maxillary bone, we penetrated the antero-medial aspect of maxillary sinus. The

154 entrance was further enlarged using a Kerrison Rongeurs and/or drilling burr. When operating

155 space was not easily accessible and a wider working area was needed, we drilled out part of the

156 anterior maxillary wall to make way extent our surgical fulcrum laterally. All the diseased

157 mucosa as well as neoplasms were removed. The underlying hyperplastic bones were drilled

158 out and cauterized electrically in patients with sinonasal papillomas (Healy et al., 2016).

159 Additionally, the medial bony compartment was taken out altogether when the lesions were

160 closely attached to the natural ostium and/or medial aspect of maxillary sinus. For

161 inflammatory lesions, much of the underlying sinus mucosa was preserved as possible while the 
162 nidus were meticulously trimmed. The IT-NLD flap was re-draped onto its primary position

163 suturing the incision site with 4-O vicryl. In some patients, an inferior meatotomy was created

164 to facilitate future observation during following visits (Fig. 1).

165

166

167

168

169

170

171

172

173

174

175

176

177

178

179

180

181

182

183

184

185

\section{Statistical analyses}

Categorical variables were expressed as frequency and percentage. Descriptive parametric data were expressed using mean, standard deviation (SD), and range. We used median and interquartile range (IQR) to express subjective metrics (VAS and SNOT-22 included). Their interval changes between different groups were tested using Wilcoxon signed rank test. The differences between two groups were tested using Mann Whitney $U$ test. P-value $<0.05$ was considered significant. All statistical operations were performed using IBM SPSS 22.0 statistical software (IBM Corp. Released 2013. IBM SPSS Statistics for Windows, Version 22.0. Armonk, NY: IBM Corp.c., Chicago, IL, USA).

\section{Results}

\section{Patients Characters}

A total of 21 patients (16 males and 5 females) with 22 lesion sites received surgical procedures utilizing the endonasal endoscopic prelacrimcal recess approach (EPLA) at our two hospitals. The mean (standard deviation [SD]) age at diagnosis was 51.7 (14.5) years (range, 17 to 81 years) and mean follow-up after surgery was 12.7 months (range, 1.4 to 41.5 months). Fourteen patients underwent these procedures as primary surgeries; the other seven received these procedures as revisions (Table 1$)$. The other procedures included septoplasty $(n=2)$, endoscopic sinus surgery $(n=14)$, and inferior meatotomies $(n=3)$. Inferior meatotomies were created for facilitating postoperative investigation in 2 patients with diffuse papilloma and in 1 
186 patient with trauma-related maxillary mucocele. Five $(71.4 \%)$ of these seven revision patients 187 received at least two interventions (Table 1 ). As can be seen in Table 1, a summary of initial 188 presentations, the most frequent symptoms were nasal obstruction and nasal discharge 189 followed by blood streaks within nasal drainages/blood tinged sputum. The duration of 190 symptoms ranged from 30 days to 72 months (mean [SD]): 17.2 [24.2] months).

191

192 Pathologies and Imaging Features

193 Patient characteristics and disease features are summarized in Table 1. Three of the 194 patients (14.3\%) had inflammatory sinonasal disorders (2 primary chronic rhinosinusitis and 1 195 recurrent bilateral CRS with nasal polyps). Eleven patients had benign neoplastic lesions within 196 the maxillary sinus. Of these eleven patients, nine patients ( $81.8 \%$; 8 unilateral, 1 bilateral) had 197 sinonasal papillomas (9 inverted, 1 exophytic) (Fig. 2A), one had a sinonasal organized 198 hematoma, and the other had a cavernous hemagioma. Three patients had odontogenic cysts, 199 including 2 patients with comorbid maxillary ectopic teeth, Fig. 2B). Within the remaining four 200 patients, two patients presented trauma-related disorders (an orbital fracture requiring medial 201 and inferior orbital repair as seen in Fig. 2C and a maxillary mucocele that developed decades 202 after facial trauma) and the other two patients with individual malignancies (lacrimal duct 203 adenoid cystic carcinoma and recurrent buccal cancer with involvement to the infratemporal 204 fossa) both underwent pathology obtainment by EPLA. The extent of disease and locations of 205 the papillomas within maxillary sinus can be found in Table 2. 206 (range, 19 to $34.9 \mathrm{~mm}$ ). Antero-posterior diameter of three diseased sides is greater than $7 \mathrm{~mm}$ whereas the measurement of the remaining 19 sides ranged from 3- to 7-mm. We did not find 
210 difference of modified-Lund-Mackay scores between patients with sinonasal papillomas and

211 chronic rhinosinusitis (3.0 [IQR 2.0-3.5] vs. 4.0 [IQR 4.0-4.0], $\mathrm{P}=0.064)$. The mean (SD) values

212 for pre-treatment SNOT-22 and VAS are 32.6 (22.1) and 6.7 (3.4), respectively. Patients who did

213 not have papillomas reported statistically higher VAS ratings and SNOT-22 scores than those

214 with papillomas (Table 3).

215

216 Outcomes and Complication Profiles

217 Within these 21 patients, 5 patients did no complete subjective outcome measurements

218 (SNOT-22 and VAS). Finally, 16 patients were enrolled in functional outcomes analysis

219 measured at the last clinical visit after primary EPLAs. There were no significant differences in

220 post-treatment patient-reported outcomes between those with sinonasal papillomas and those

221 with other pathologies (Table 3). Patients who did not have papillomas reported significantly

222 improved VAS ratings and SNOT-22 scores (Table 3, both P-values $<0.05$ ), while individuals with

223 papillomas also reported improved VAS ratings and SNOT-22 scores, though without statistical

224 significance (Table 3). There was no disease recurrence during the follow-up period.

225

226 Eleven patients had 13 adverse events. Papilloma patients had a significantly higher

227 incidence of post-operative complications, compared to patients with other pathologies ( $n=11$

228 vs. $n=2)$. The most commonly seen complication was paresthesia $(n=11)$ of cheek and tooth,

229 and all symptoms of this complication resolved within 6 months. Patients with AP diameter

230 ranged from 3- to 7- $\mathrm{mm}$ tend to have higher prevalence of surgery-associated neural sequels,

231 compared with individuals with AP diameter greater than $7 \mathrm{~mm}(10 / 19,52.6 \%$ vs. 1/3, 33\%,

232 respectively), though of no statistical significance $(P=1.00)$. One patient had delayed wound

233 healing resulting from a prior submucosal turbinectomy allegedly caused by post-surgical 
234 scarring. Additionally, one patient developed epistaxis soon after the surgery. No nasal lacrimal

235 ducts or negative aesthetic issues (i.e., nasal alar collapse/soft tissues depressions) were found.

236

237 Discussion

238 This study found that EPLA-treated patients reported improved VAS ratings SNOT-22

239 scores over time, with mean improvements of 4.2 and 17.6 points, respectively. These scores

240 suggest significant improvement in quality of life after EPLAs. Although we encountered

241 treatment-associated adverse events, most of them are paresthesia occurred in inverted

242 papilloma cases. However, all adverse events were all manageable and the sensory deficits

243 subsided within 6 months. These findings suggest that the endoscopic prelacrimal recess

244 approach can be used safely and effectively to treat a variety of maxillary sinus pathologies.

245

246

In the surgical treatment of diseases originating from different sites of the maxillary sinus,

247 the endoscopic pre-lacrimal approach makes possible the management of almost any aspect of

248 internal linings. Especially the anterior and inferior aspect (e.g., alveolar recess and PLR), which

249 are locations that traditional surgical corridors can hardly access (Nakamaru et al.,2010; Zhou et

250 al., 2013; Zhou et al., 2016; Lee et al., 2019; Zhou et al., 2018; Yu et al., 2018; Lin, Lin \& Yeh,

251 2018). In select cases in which disease is located far laterally, surgeons can drill the pyriform

252 aperture and/or part of anterior maxillary wall to extend surgical fulcrum (Turri-Zanoni et

253 al.,2017; Nakamaru et al., 2010; Zhou et al., 2013).

254

255

One recent study has reported that operations employing EPLA to treat maxillary or retro-

maxilla lesions can achieve outcomes comparable to those achieved by traditional open

257 approaches performed by experienced hands (Zhou et al.,2016; Lee et al., 2019). However, 
258 before we try to implement this technique more widely, some of its disadvantages need to be 259 understood. The first being that the inferior turbinate-nasal lacrimal duct mucosal flap is re260 draped onto its original position, the anterior half of the maxillary sinus (especially the alveolar 261 recess inferiorly and zygomatic recess laterally) is difficult to be evaluated even with the use of 262 flexible endoscopy post-operatively (Zhou et al.,2018). Therefore, additional inferior 263 meatotomy may be necessary for full-filed surveillance for patients with high risk of disease 264 recurrence (Zhou et al.,2018). Further, post-surgical debridement and saline delivery are less 265 convenient in comparison with standard endoscopic medial maxillectomy (Turri-Zanoni et 266 al.,2017). Still another challenge is that EPLA must be very carefully performed when treating 267 neoplasms involving or infiltrating the inferior turbinate, nasolacrimal complex or medial wall of 268 the maxillary sinus (Nakamaru et al.,2010; Zhou et al., 2013). In addition, overzealous 269 manipulation of mucosa within alveolar recess should be avoided to prevent inadvertent 270 trauma to the minor branches of anterior superior alveolar nerve (branches from the

271 infraorbital nerve running within the maxilla). Care must also be taken to avoid dental injury 272 because first and second molar tooth are dehiscent into the maxillary sinus, which has been 273 reported to occur in $2 \%$ of normal population (Coleman et al.,2005).

274

275 Initially EPLA for neoplastic lesions were designed to provide a corridor to allow the 276 management of lesions within infratemporal and pterygopalatine fossa tumors (Zhou et 277 al.,2016). Recently, its use has been extended to the treatment of maxillary neoplasms, mostly 278 sinonasal inverted papillomas (SNIPs) (Zhou et al.,2013; Lee et al., 2019; Zhou et al., 2018; Yu et 279 al., 2018). The benefits of EPLAs over standard endoscopic maxillectomies when treating SNIPs 280 include its ability to preserve as many normal structures as possible (Turri-Zanoni et al.,2017), 281 which makes wound healing faster, and its ability to avoid alter turbulent nasal airflow due to 
282 preserve of inferior turbinate (Chen et al.,2019). It has also been associated with less lacrimal 283 pathway obstruction and fewer thick crusting because more of the epithelium is preserved 284 (Hildenbrand, Weber \& Brehmer, 2011). In their multicenter retrospective study, Zhou (2018) 285 reported recurrence rates in 71 Krous staged III SNIPs patients treated with EPLAs to be similar 286 to those reported by Lombardi (2011) performing the largest endoscopically cohort study to 287 date. However, patients in Zhou (2018) were spared from post-operative mucocele 288 developments and negative naso-lacrimal complex sequelae (Lombardi et al.,2011; Zhou et al., 289 2018). Bertazzoni (2017), using extended endoscopic maxillectomies exclusively, also found 290 significantly greater number for sensory deficits and aesthetic problems than Zhou (2018) 291 reported. Three studies of EPLA SNIPs resections in relatively small patient groups have recently 292 reported EPLAs to result in favorable local control rates, though they did not investigate post293 surgery quality-of-life (Lee et al.,2019; Yu et al., 2018; Lin, Lin \& Yeh, 2018). Our eight sinonasal 294 papillomas patients reported a median SNOT-22 score of 8 after resection, slightly lower than 295 the scores reported by a previously reported case series (Harrow \& Batra, 2013). Although 296 statistical significance was not reached in our study, our results suggest improvements quality 297 of life metrics after EPLA in these patients.

298

299 300 301 302 303 304 305

The proposed mechanisms for relatively higher incidence of transient neural sequel in our sinonasal papilloma patients are illustrated as the follows. The first is the majority of our study population has type II prelacrimal recess (antero-posterior diameter ranged from 3- to 7- $\mathrm{mm}$ ) (Simmen et al., 2017). In an imaging study investigating the feasibility of EPLAs, Simmen (2017) concluded more bone removal and probable translation tear sac are needed in type II subjects in comparison to those with type III ( $A P>7 \mathrm{~mm}$ ). More bone removal might imply higher probabilities for nerve insults. Another is our exclusive performance of bone drilling on tumor 
306 bed (Healy et al., 2016). We hypothesize the direct and indirect nerve injuries due to osculating

307 and heating effects by drillings may be responsible for the relative higher incidence for

308 transient sensory deficit. As for residual inferior meatotomy, Preti (2019) proposed an

309 innovative method by performing mucosal incision and osteotomy at different levels to 310 minimize such an adverse event.

311

Some studies have reported greater/faster symptoms reduction and less disease

313 recurrence following surgeries compared with appropriate pharmaceutical managements alone

314 in certain subgroups of chronic rhinosinusitis (Fokken et al.,2019; Alsharif et al., 2019; Wang,

315 Gullung \& Schlosser, 2011). However, the appropriateness of primary EPLAs to treat lesions due

316 to inflammation in rhinosinusitis has not been addressed. Our reason for extending the use of

317 EPLA to inflammatory maxillary disease is that EPLAs can offer intuitive viewing and more

318 maneuvering space by zero degree endoscope. Thus, EPLAs may save more time than MMAs

319 without additional complications, especially when treating cases in which inflammatory polyps

320 full of maxillary sinus. In these cases, stripping lesions arising from the anterior and/or medial

321 aspect of maxillary sinus (e.g., alveolar or prelacrimal recess) present an obstacle for standard

322 MMA approach (Turri-Zanoni et al.,2017; Kashlan \& Craig, 2018). Nonetheless, further studies

323 are needed to elucidate whether patients with inflammatory maxillary disease undergoing

324 EPLAs have results comparable with those treated with conventional MMAs.

325

326 This study has some limitations. One limitation is its relatively small sample size and a lack

327 of a control cohort treated with other surgical strategies. This made further comparisons of

328 multiple endpoints among different cohorts with maxillary lesions difficult. Another limitation is

329 that because we only had access to post-operative outcomes approximate six months later, we 
330 could not determine whether reduced inflammation reached nadir after surgical treatment

331 using EPLA. An additional limitation is that we remain unclear regarding the altered extent of

332 nasal airway resistance and maxillary sinus mucociliary clearance post-operatively. Future

333 investigations are needed to verify long-term rhinologic manifestations. Still another is we did

334 not analyze influencing factors (e.g., laterality [Beswick et al.], surgical extent [Ayoub et al.],

335 comorbid rhinitis, and concomitant surgical procedures, etc) in the current study. Considering

336 their probable impacts on VAS rating and SNOT-22 scores, future studies are warranted to test

337 their significance in the framework of EPLAs.

338

339 Conclusion

340 In conclusion, we found that in addition to tumors, EPLAs can be used to safely and

341 efficaciously treat various lesions within the maxillary sinus. This approach might be used to

342 replace invasive or time-consuming surgical strategies currently used to manage certain

343 pathologies. We found most common adverse event to be transient cheek/tooth numbness but

344 they usually subsided within 6 months. Thus, with adequate patient counseling, symptoms

345 reduction and improvements in life quality can be expected after receiving surgical treatment

346 of maxillary sinus lesions using EPLAs.

347

348 Abbreviations

$349 \operatorname{EPLA}(\mathrm{s})$, endoscopic prelacrimal recess approache(s)

350 SNOT-22, 22-item Sino-Nasal Outcomes Test

351 VAS, visual analogue scales

352 MMA, middle meatal antrostomy

353 PLR, prelacrimal recess 
354 AJCC, American Joint Committee on Cancer

355 AP, antero-posterior

356 NLD, nasal lacrimal duct

357 IT-NLD, inferior turbinate-nasolacrimal duct

358 SD, standard deviation

359 IQR, interquartile range

360 SNIP(s), sinonasal inverted papilloma(s)

361

362 Conflict of Interest Disclosures

363 None of the authors declare any conflict of interest, financial or otherwise.

364

365 Compliance with Ethical Standards

366 The protocol of this study was approved by the institutional review board of Kaohsiung Chang

367 Gung Memorial Hospital (IRB No.: 201900695B0).

368 
369

370

371

372

373

374

375

376

377

378

379

380

381

382

383

384

385

386

387

388

389

390

391

392

\section{References}

1. Tomenzoli D, Castelnuovo P, Pagella F, Berlucchi M, Pianta L, Delù G, Maroldi R, Nicolai P. 2014. Different endoscopic surgical strategies in the management of inverted papilloma of the sinonasal tract: experience with 47 patients. Laryngoscope 114:193-200.

2. Coleman H, Meer S, Altini M, Reyneke J, Becker P. 2005. Maxillary sinus pathology in 119 patients--a histopathologic study. SADJ 60:142-145.

3. Kennedy DW, Adappa ND. 2011. Endoscopic maxillary antrostomy: not just a simple procedure. Laryngoscope 121:2142-2145.

4. Turri-Zanoni M, Battaglia P, Karligkiotis A, Lepera D, Zocchi J, Dallan I, Bignami M, Castelnuovo P. 2017. Transnasal endoscopic partial maxillectomy: Operative nuances and proposal for a comprehensive classification system based on 1378 cases. Head Neck 39:754766.

5. Wormald PJ, Ooi E, van Hasselt CA, Nair S. 2003. Endoscopic removal of sinonasal inverted papilloma including endoscopic medial maxillectomy. Laryngoscope 113:867-873.

6. Lombardi D, Tomenzoli D, Buttà L, Bizzoni A, Farina D, Sberze F, Karligkiotis A, Castelnuovo P, Nicolai P. 2011. Limitations and complications of endoscopic surgery for treatment for sinonasal inverted papilloma: a reassessment after 212 cases. Head Neck 33:1154-1161.

7. Bertazzoni G, Accorona R, Schreiber A, Pietrobon G, Karligkiotis A, Fazio E, Castelnuovo P, Nicolai P. 2017. Postoperative long-term morbidity of extended endoscopic maxillectomy for inverted papilloma. Rhinology 55:319-325.

8. Nakamaru Y, Furuta Y, Takagi D, Oridate N, Fukuda S. 2010. Preservation of the nasolacrimal duct during endoscopic medial maxillectomy for sinonasal inverted papilloma. Rhinology 48:452-456. 
393 9. Zhou B, Han DM, Cui SJ, Huang Q, Wang CS. 2013. Intranasal endoscopic prelacrimal recess 394 approach to maxillary sinus. Chin Med J (Engl) 126:1276-1280.

395

396

397

398

399

400

401

402

403

404

405

406

407

408

409

410

411

412

413

414

415

416

10. Zhou B, Huang Q, Shen PH, Cui SJ, Wang CS, Li YC, Yu ZK, Chen XH, Ye T. 2016. The intranasal endoscopic removal of schwannoma of the pterygopalatine and infratemporal fossae via the prelacrimal recess approach. J Neurosurg 124:1068-1073.

11. Lee JJ, Ahmad Z AM, Kim D, Ryu G, Kim HY, Dhong HJ, Chung SK, Hong SD. 2019. Comparison Between Endoscopic Prelacrimal Medial Maxillectomy and Caldwell-Luc Approach for Benign Maxillary Sinus Tumors. Clin Exp Otorhinolaryngol 12:287-293.

12. Zhou B, Huang Q, Sun J, Li X, Zhang W, Cui S, Shen PH, Wang C, Huang Z, Dong Y, Liang N. 2018. Resection of Inverted Papilloma of the Maxillary Sinus via a Prelacrimal Recess Approach: A Multicenter Retrospective Analysis of Surgical Efficacy. Am J Rhinol Allergy 32:518-525.

13. Yu QQ, Guan G, Zhang NK, Zhang XW, Jiang Y, Lian YY, Liu TT, Jiang XD, Li N. 2018. Intranasal endoscopic prelacrimal recess approach for maxillary sinus inverted papilloma. Eur Arch Otorhinolaryngol 275:2297-2302.

14. Lin YT, Lin CF, Yeh TH. 2018. Application of the endoscopic prelacrimal recess approach to the maxillary sinus in unilateral maxillary diseases. Int Forum Allergy Rhinol 8:530-536.

15. Snidvongs K, Dalgorf D, Kalish L, Sacks R, Pratt E, Harvey RJ. 2014. Modified Lund Mackay Postoperative Endoscopy Score for defining inflammatory burden in chronic rhinosinusitis. Rhinology 52:53-59.

16. Krouse JH. Development of a staging system for inverted papilloma. 2000. Laryngoscope 110:965-968.

17. Kashlan K, Craig J. 2018. Dimensions of the medial wall of the prelacrimal recess. Int Forum Allergy Rhinol 8:751-755. 
417 18. DeConde AS, Mace JC, Alt JA, Rudmik L, Soler ZM, Smith TL. 2015. Longitudinal 418 improvement and stability of the SNOT-22 survey in the evaluation of surgical management 419 for chronic rhinosinusitis. Int Forum Allergy Rhinol 5:233-239.

420 19. Healy DY, Chhabra N, Metson R, Gray ST. 2016. Surgical risk factors for recurrence of 421 inverted papilloma. Laryngoscope 126:796-801.

422 20. Chen XB, Leong SC, Lee HP, Chong VF, Wang DY. 2010. Aerodynamic effects of inferior 423 turbinate surgery on nasal airflow--a computational fluid dynamics model. Rhinology 48:394-400.

21. Hildenbrand T, Weber RK, Brehmer D. 2011. Rhinitis sicca, dry nose and atrophic rhinitis: a review of the literature. Eur Arch Otorhinolaryngol 268:17-26.

22. Harrow BR, Batra PS. 2013. Sinonasal quality of life outcomes after minimally invasive resection of sinonasal and skull-base tumors. Int Forum Allergy Rhinol 3:1013-1020.

23. Simmen D, Veerasigamani N, Briner HR, Jones N, Schuknecht B. 2017. Anterior maxillary wall and lacrimal duct relationship - CT analysis for prelacrimal access to the maxillary sinus. Rhinology 1;55:170-174.

24. Preti A, Turri-Zanoni M, Arosio AD, Castelnuovo P, Battaglia P. 2019. A Surgical Variant of the Pre-Lacrimal Approach to the Maxillary Sinus. Iran J Otorhinolaryngol 31:327-328.

25. Fokkens W, Desrosiers M, Harvey R, Hopkins C, Mullol J, Philpott C, Alobid I, AnselmoLima WT, Bachert C, Baroody F, Bernal-Sprekelsen M, von Buchwald C, Cervin A, Cohen N, Constantinidis J, De Gabory L, Douglas R, Gevaert P, Hafner A, Hellings P, Joos G, Kalogjera L, Kern R, Knill A, Kocks J, Landis BN, Limpens J, Lebeer S, Lourenco O, Matricardi PM, Meco C, O Mahony L, Reitsma S, Ryan D, Schlosser R, Senior B, Smith T, Teeling T, Tomazic PV, Toppila-Salmi S, Wang DY, Wang D, Zhang L, Lund V. 2019. EPOS2020: development 
$441 \quad 57: 162-168$.

442 26. Alsharif S, Jonstam K, van Zele T, Gevaert P, Holtappels G, Bachert C. 2019. Endoscopic 443 Sinus Surgery for Type-2 CRS wNP: An Endotype-Based Retrospective Study. Laryngoscope $444 \quad$ 129:1286-1292.

445 27. Wang EW, Gullung JL, Schlosser RJ. 2011. Modified endoscopic medial maxillectomy for 446 recalcitrant chronic maxillary sinusitis. Int Forum Allergy Rhinol 1:493-497.

447 28. Beswick DM, Mace JC, Chowdhury NI, Alt JA, Hwang PH, DeConde AS, Smith TL. 2017. 448 Comparison of surgical outcomes between patients with unilateral and bilateral chronic $449 \quad$ rhinosinusitis. Int Forum Allergy Rhinol 7:1162-1169.

450 29. Ayoub N, Walgama E, Thamboo A, Chitsuthipakorn W, Patel ZM, Nayak JV, Hwang PH. 451 2019. Correlation between extent of sinus surgery, radiographic disease, and postoperative 452 outcomes. Rhinology 31. doi: 10.4193/Rhin19.213.

453

454

455

456

457

458

459

460

461

462 Figure Legends

463 Figure 1.

464 Inferior meatotomy was created to facilitate post-operative follow-up in inverted papilloma 
465 patients with risk of recurrence.

466

467 Figure 2.

468 Endoscopic pre-lacrimal approach for (A) left maxillary sinus inverted papilloma (B) left

469 orbital floor defect repair with medpor (C) right maxillary sinus ectopic tooth removal. 


\section{Table 1 (on next page)}

Demographic characteristic of 21 patients with underwent endonasal endoscopic prelacrimal recess approach 
1 Table 1 Demographic characteristic of 21 patients with underwent endonasal endoscopic

2 prelacrimal recess approach

\begin{tabular}{|c|c|c|}
\hline Variables & $\begin{array}{l}\text { Sinonasal } \\
\text { papilloma } \\
n=9\end{array}$ & $\begin{array}{l}\text { Non papilloma } \\
\mathrm{n}=12\end{array}$ \\
\hline Age, mean (SD) & $57.0 \pm 15.4$ & $47.8 \pm 13.7$ \\
\hline \multicolumn{3}{|l|}{ Gender, n (\%) } \\
\hline male & $7(77.8)$ & $9(75)$ \\
\hline female & $2(22.2)$ & $3(25)$ \\
\hline \multicolumn{3}{|l|}{ Initial symptoms, n (\%) } \\
\hline nasal airway obstruction & $4(44.4)$ & $3(25)$ \\
\hline $\begin{array}{l}\text { nasal discharge/discolored post nasal } \\
\text { drips }\end{array}$ & $2(22.2)$ & $6(50)$ \\
\hline facial\&head pain/pressures & 0 & $5(41.7)$ \\
\hline hyposmia/anosmia & 0 & $1(8.3)$ \\
\hline blood tinged sputum/rhinorrhea & $5(55.6)$ & $1(8.3)$ \\
\hline ophthalmologic manifestations & 0 & $2(16.6)$ \\
\hline asymptomatic & $1(11.1)$ & 0 \\
\hline \multicolumn{3}{|l|}{ CT Lund-Mackay scores, mean (SD) } \\
\hline maxillary sinus (modified) & $3.0 \pm 0.7$ & $2.9 \pm 1.2$ \\
\hline total & $5.7 \pm 6.8$ & $4.6 \pm 5.0$ \\
\hline \multicolumn{3}{|l|}{ Measured nasolacrimal complex } \\
\hline \multicolumn{3}{|l|}{ parameters, mean (SD) } \\
\hline antero-posterior dimension (mm) & $5.2 \pm 1.3$ & $5.6 \pm 1.5$ \\
\hline height (mm) & $26.4 \pm 3.7$ & $27.3 \pm 5.0$ \\
\hline Revision procedures, n (\%) & $4(44.4)$ & $3(25)$ \\
\hline \multicolumn{3}{|l|}{ Complications, n (\%) } \\
\hline transient dental/cheek numbness & $9(100)$ & $2(16.7)$ \\
\hline epistaxis & $1(11.1)$ & 0 \\
\hline delayed wound healing & $1(11.1)$ & 0 \\
\hline
\end{tabular}

3

$\mathrm{CT}$, computed tomography; SD, standard deviation

4 
Table 2 (on next page)

Characteristic features of patients with sinonasal papilloma 
Table 2 Characteristic features of patients with sinonasal papilloma

Patient number Gender Age Pretreatment VAS Pretreatment SNOT-22

$\begin{array}{cccc}1 & M & 59 & 8 \\ 2 & M & 56 & 1 \\ 3 & M & 30 & \text { N/A } \\ 4 & F & 49 & 3 \\ 5 & M & 44 & 5 \\ 6 & M & 71 & 0 \\ 7 & F & 54 & 10 \\ 8 & M & 81 & 4 \\ 9 & M & 69 & 6\end{array}$

8
1
3
3
5
0
0
4
6

\section{(17}

17

Disease extent within maxillary sinus (disease side)

Follow-up
time, months

$M, P(L)$

$M, P(L)$

$M, A, L(R)$

$P, L(L)$

$\mathrm{I}, \mathrm{M}(\mathrm{L})$

$A, I(L)$

A (B)

ALL (L)

$A, L, P(R)$
Previous

operation

17 N

$\mathrm{N}$

$37 \quad \mathrm{Y}$

$7 \quad \mathrm{~N}$

$\begin{array}{ll}7 & N \\ 7 & N\end{array}$

$42 \quad \mathrm{~N}$

$41 \quad Y$

20

1 Abbreviation: VAS, visual analogue scales; SNOT-22, 22-item Sino-Nasal Outcomes Test; M, medial; P, posterior; L, lateral; A, anterior;

2 I, inferior; N/A, not available; $N$, no; $Y$, yes 


\section{Table 3 (on next page)}

Outcomes of patients treated with endoscopic prelacrimal recess approach 
Table 3 Outcomes of patients treated with endoscopic prelacrimal recess approach

\begin{tabular}{lccc}
\hline & Pre-treatment & Post-treatment & \\
\hline & VAS, median (IQR) & VAS, median (IQR) & P-value \\
Papillomas & $4.5(1.5-7.5)$ & $1.5(0-3.5)$ & 0.061 \\
Non-papillomas & $8.8(7.3-10.0)$ & $1.8(0.3-3.2)$ & 0.012 \\
P-value & 0.050 & 0.867 & P-value \\
Papillomas & SNOT-22, median (IQR) & SNOT-22, median (IQR) & 0.176 \\
Non-papillomas & $17.5(5.8-25.3)$ & $8.0(3.3-11.0)$ & 0.012 \\
P-value & $37.5(26.5-50.3)$ & $13.5(10.8-28.8)$ &
\end{tabular}

Abbreviation: VAS, visual analogue scales; SNOT-22, 22-item Sino-Nasal Outcomes Test; IQR, interquartile range 


\section{Figure 1}

Inferior antrostomy is created after pre-lacrimal approach combined with medial maxillectomy.

Inferior antrostomy was created to facilitate post-operative follow-up in inverted papilloma patients with risk of recurrence.

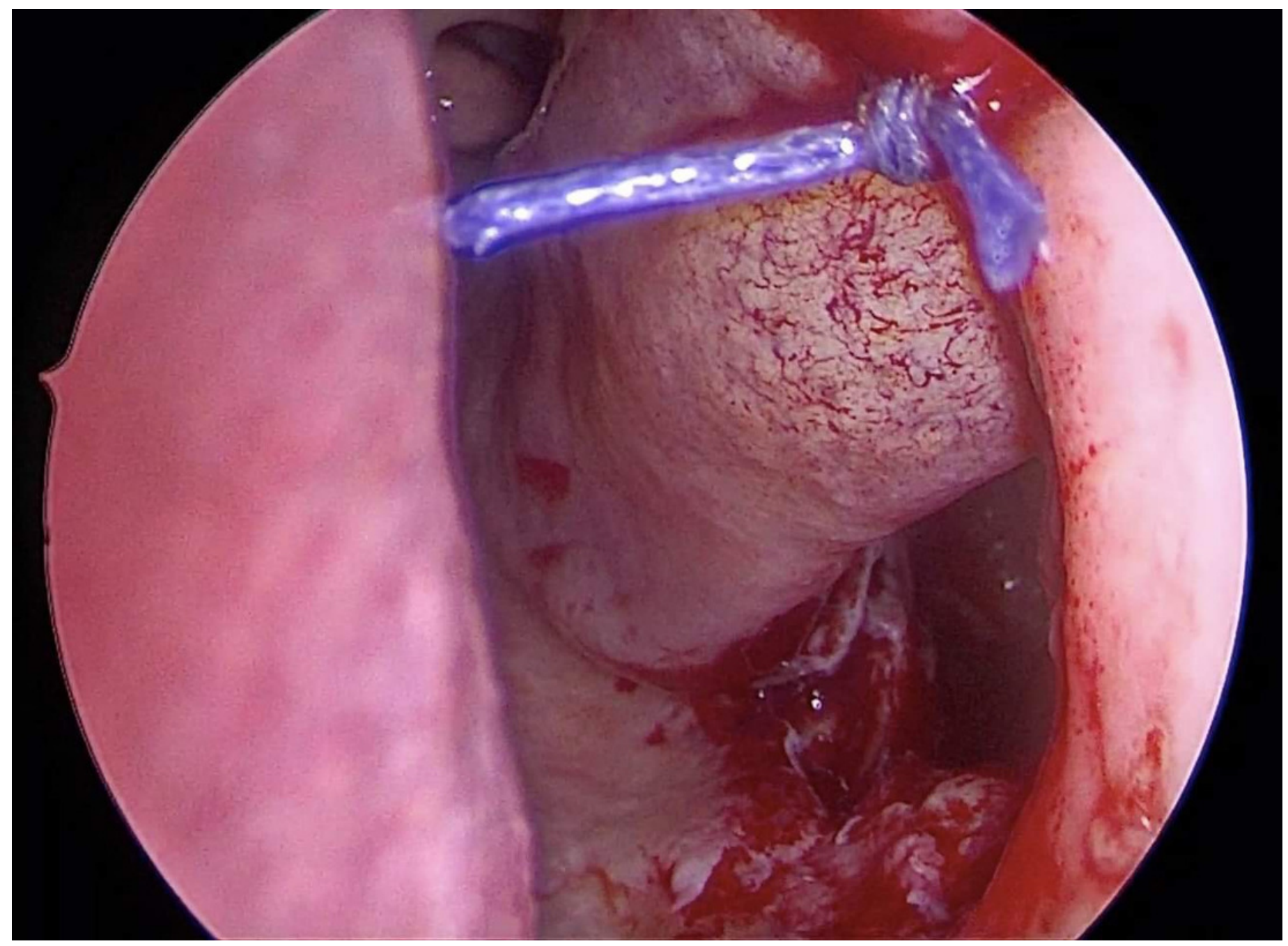




\section{Figure 2}

Pre-lacrimal approach is used to treat different maxillary sinus pathologies.

Endoscopic pre-lacrimal approach for (A) left maxillary sinus inverted papilloma (B) left orbital floor defect repair with medpor $(\mathbf{C})$ right maxillary sinus ectopic tooth removal.

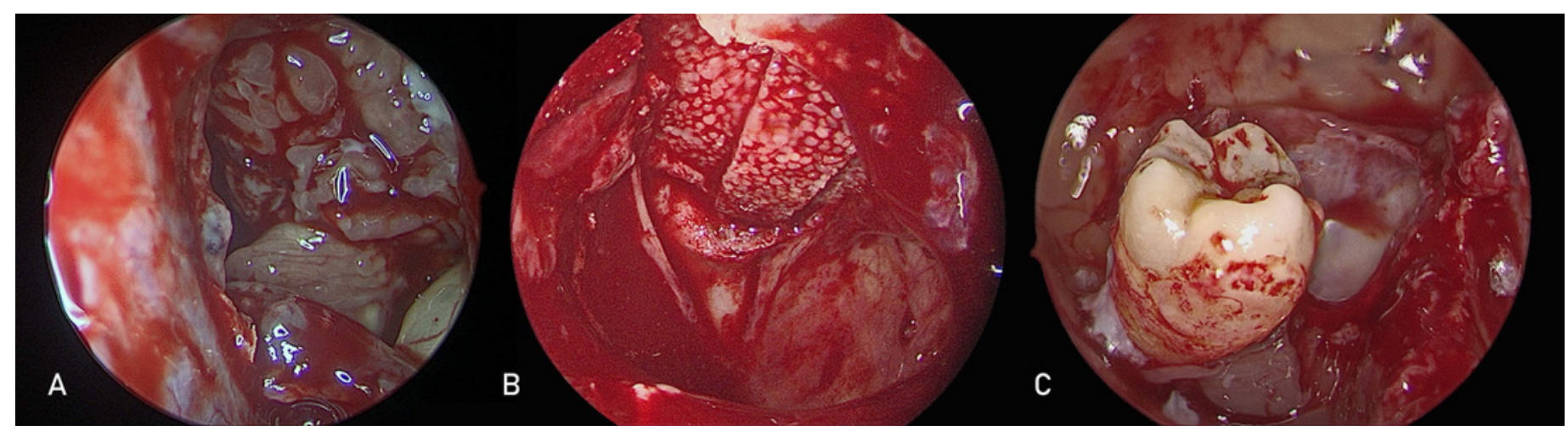

